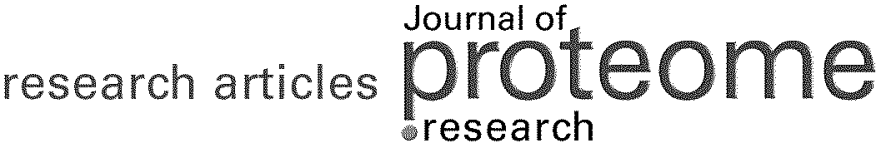

\section{Nuclear Magnetic Resonance (NMR) Study of the Effect of Cisplatin on the Metabolic Profile of MG-63 Osteosarcoma Cells}

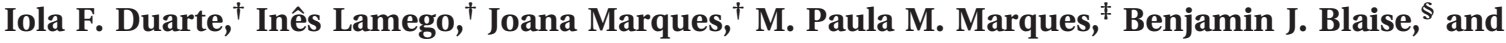 \\ Ana M. Gil*, ${ }^{*}$ \\ CICECO-Departmento de Química, Campus Universitário de Santiago, Universidade de Aveiro, \\ 3810-193 Aveiro, Portugal, R\&D Unit "Molecular Physical-Chemistry", Department of Life Sciences, Faculty of \\ Science and Technology, University of Coimbra, Portugal, and Université de Lyon, Centre de RMN à Très Hauts \\ Champs, 5 rue de la Doua, 69100 Villeurbanne, France
}

Received June 21, 2010

\begin{abstract}
In the present study, ${ }^{1} \mathrm{H}$ HRMAS NMR spectroscopy was used to assess the changes in the intracellular metabolic profile of MG-63 human osteosarcoma (OS) cells induced by the chemotherapy agent cisplatin (CDDP) at different times of exposure. Multivariate analysis was applied to the cells spectra, enabling consistent variation patterns to be detected and drug-specific metabolic effects to be identified. Statistical recoupling of variables (SRV) analysis and spectral integration enabled the most relevant spectral changes to be evaluated, revealing significant time-dependent alterations in lipids, choline-containing compounds, some amino acids, polyalcohols, and nitrogenated bases. The metabolic relevance of these compounds in the response of MG-63 cells to CDDP treatment is discussed.
\end{abstract}

Keywords: osteosarcoma • MG-63 cells • cisplatin • cancer • cancer therapy • metabonomics • NMR spectroscopy

\section{Introduction}

Osteosarcoma (OS) is the most common primary malignant bone cancer occurring in childhood. The introduction of systemic chemotherapy in the 1970s markedly improved longterm survival from 15 to $70 \%$ for patients with localized disease. ${ }^{1}$ However, the prognosis is still poor for the subset of patients with metastatic or recurrent disease, whose chances of relapse-free survival are less than $20 \%$. Currently, the most widely used chemotherapy protocols combine cisplatin, doxorubicin and methotrexate, and significant efforts to enhance the efficacy of these agents and to develop new drugs are underway. $^{2}$

Improved treatment management and outcome also depend on the ability to monitor and understand the organism's response to the drugs administered. Therefore, it is important to develop methodologies with the ability to provide detailed and accurate information on drug effects produced locally and at the systemic level. Metabonomics (or metabolomics) has an emerging role in this context, as the metabolome of cancer cells or living organisms is likely to show changes upon the response to an anticancer drug. ${ }^{3}$ By correlating metabolic variations to drug exposure conditions (e.g., treatment duration and dose), this approach may enable biomarkers for drug toxicity and efficacy to be found, thus providing important insights into the drug's mechanism of action, as well as helping to adjust and optimize treatment plans in a clinical setting. Nuclear magnetic

\footnotetext{
* To whom correspondence should be addressed. E-mail: agil@ua.pt

${ }^{\dagger}$ Universidade de Aveiro.

‡ University of Coimbra.

§ Université de Lyon.
}

resonance (NMR) studies have shown great potential for assessing tumor response to anticancer agents and providing insights into the mechanisms of action and resistance of drugs. ${ }^{3,4}$ In spite of being inherently insensitive, NMR allows for the direct analysis of cells, tissues and biofluids, in a nondestructive, quantitative and reproducible manner. Information is obtained simultaneously for a wide range of compounds involved in different biochemical pathways, thus enabling comprehensive metabolic pictures and hidden biological events to be unveiled. Moreover, the possible extrapolation of biomarkers detected initially in tissues or cells by highresolution NMR to in vivo detection by clinical MR scanners makes this approach exquisitely valuable for the development of methods for noninvasive assessment of therapeutic responses.

In spite of limitations such as oversimplicity and dosimetry mismatch compared to in vivo conditions, in vitro cell studies are still a widely used approach for investigating the effects of anticancer drugs by NMR metabonomics, constituting an important first step toward understanding the biological response to an external agent in the body. The application of NMR metabolic profiling to cultured cells has been explored in a wide range of situations, for example, study of cell cycle stage, genetic characteristics, disease, programmed cell death and response to radiation or drug exposure. ${ }^{5-8}$ The metabolome of human osteosarcoma (OS) cells has been studied by NMR in regard to culture type ${ }^{9}$ and evaluation of the changes induced by ionizing radiation. ${ }^{10-12}$ In the first study, ${ }^{1} \mathrm{H}$ NMR of MG-63 cell suspensions and extracts showed that cell organization in monolayer and in spheroids results in different metabolic profiles, affecting mainly energy production and possibly lipid metabolism, whereas the subsequent studies 
highlighted dose and time-related metabolic changes in MG63 spheroids subjected to radiation treatment. Recently, another human OS cell line, 143B, has been compared to hepatocarcinoma cells (HepG2), with distinct mitochondrial oxidative phosphorylation background, and differential responses to anticancer agent chloroethylnitrosourea (CENU) were assessed through respiratory and energy production parameters together with NMR metabolic profiling. ${ }^{13}$ In addition, many different anticancer drugs have been investigated through NMR analysis of other types of cultured cells, mostly in the form of cell suspensions ${ }^{14-17}$ or extracts. ${ }^{18-22}$ High resolution magic angle spinning (HRMAS) NMR spectroscopy, which is well established in tissue analysis, ${ }^{23,24}$ has been shown to enable well-resolved spectra to be obtained for intact or lysed cells $^{25}$ and to provide valuable information on both lipid and small metabolite changes upon treatment with anticancer drugs of endometrial, ${ }^{26}$ melanoma, ${ }^{27}$ colon carcinoma, ${ }^{28}$ hepatocarcinoma and osteosarcoma ${ }^{13}$ cells.

In the present study, ${ }^{1} \mathrm{H}$ HRMAS NMR spectroscopy was used to assess the changes in the intracellular metabolic profile of MG-63 human OS cells induced by the chemotherapy agent cisplatin (cis-diamminedichloroplatinum(II) or CDDP) at different times of exposure. Multivariate analysis (MVA) was applied to the cells spectra in order to probe for consistent variation patterns and separate the effects due to drug exposure from the confounding influence of other factors, such as the metabolic variability inherent to cell growth. Metabolite signals highlighted by statistical recoupling of variables (SRV) to be important in the cells response to CDDP were, whenever possible, integrated to provide a quantitative measurement of the variations over time, both in controls and drug-treated cells. Finally, the metabolic relevance of the compounds thus identified was discussed.

\section{Experimental Section}

Cell Growth and Exposure to Cisplatin (CDDP). MG-63 osteosarcoma cells, kindly provided by the Associate Laboratory IBMC-INEB, Portugal, were grown in Eagle's Minimum Essential Medium (MEM) supplemented with $10 \%$ heat-inactivated fetal bovine serum, $1 \mathrm{mM}$ sodium pyruvate, nonessential amino acids and antibiotics (penicillin-streptomycin 100×) and maintained at $37^{\circ} \mathrm{C}$ in a humidified atmosphere under $5 \% \mathrm{CO}_{2}$. Ninety-six hours after seeding, the cells were harvested upon addition of trypsin ( $0.05 \%$ trypsin/EDTA solution) and plated in $10 \mathrm{~cm}$ diameter Petri dishes $\left(5 \times 10^{6}\right.$ cells each). After waiting for $24 \mathrm{~h}$ for cells to adhere, the experiment was initiated $(t=$ 0 ) by adding to Petri dishes (containing $12 \mathrm{~mL}$ culture medium) $600 \mu \mathrm{L}$ CDDP stock solutions $1 \mathrm{mM}$ and $0.6 \mathrm{mM}$ to give final CDDP concentrations of 30 or $50 \mu \mathrm{M}$, respectively. Nothing was added to control cells. At different time points $(t=12,18,24$, and $48 \mathrm{~h}$ ), the adherent cells were harvested by trypsinisation, washed with phosphate buffer solution (PBS, pH 7.4), and centrifuged at $1000 \mathrm{rpm}$ for $6 \mathrm{~min}$. Lysed suspensions were then prepared as described below. Three independent assays with duplicates of each condition (time point and dosage) were performed. For NMR analysis, the duplicates were mixed, when necessary, to provide samples with a sufficiently large number of cells (above $1 \times 10^{7}$ cells) for good signal-to-noise spectra to be obtained.

Cell Sampling and Preparation. Upon washing with PBS, the cells were suspended in $1 \mathrm{~mL} P B S / \mathrm{D}_{2} \mathrm{O}(0.14 \mathrm{M} \mathrm{NaCl}, 0.0027$ $\mathrm{M} \mathrm{KCl}, 0.0015 \mathrm{M} \mathrm{KH}_{2} \mathrm{PO}_{4}, 0.0081 \mathrm{M} \mathrm{Na}_{2} \mathrm{HPO}_{4}$ in deuterated water, $\mathrm{pH}$ 7.4), centrifuged and resuspended in $40 \mu \mathrm{L} \mathrm{PBS} / \mathrm{D}_{2} \mathrm{O}$, to which $5 \mu \mathrm{L}$ PBS $/ \mathrm{D}_{2} \mathrm{O}$ containing TSP $0.25 \%$ (for chemical shift referencing) were added. Then, a 3-fold cycle of liquid nitrogen deeping and sonication was performed to obtain a suspension of lysed cells. This method has been reported for the preparation of lysed cells for NMR analysis. ${ }^{25}$ Each sample was then transferred to an NMR disposable insert with sealing cap (ca. $35 \mu \mathrm{L}$ ) and stored at $-80{ }^{\circ} \mathrm{C}$ until analysis.

Trypan Blue Exclusion Assay. At each time point, the extent of cell death was assessed using the Trypan blue exclusion assay, which yields the number of living vs dead cells thus providing a measure of cell membrane integrity. ${ }^{29}$ Briefly, cells were incubated with Trypan blue dye $(0.4 \%(\mathrm{w} / \mathrm{v})$ solution in PBS) and counted in a Neubauer-counting chamber. Cell viability was expressed as the percentage of living cells (dyeexcluding) relative to the total number of cells (mean \pm SEM, standard error of the mean), ${ }^{30}$ having allowed the $\mathrm{IC}_{50}$ value (drug concentration causing $50 \%$ of cell death, at $24 \mathrm{~h}$ ) to be estimated as $30 \mu \mathrm{M}$.

NMR Measurements. The disposable inserts containing the cell samples were placed in standard $4 \mathrm{~mm}$ MAS rotors and analyzed in a Bruker Avance spectrometer operating at 700 $\mathrm{MHz}$ for ${ }^{1} \mathrm{H}$ observation, at $277 \mathrm{~K}$, using a $4 \mathrm{~mm}$ HRMAS probe, in which the rotor containing the sample was spun at the magic angle and a $4 \mathrm{kHz}$ spinning rate. A standard 1D spectrum (pulse program "noesyprld", Bruker library) was acquired for each sample with a spectral width of $6510 \mathrm{~Hz}, 32 \mathrm{~K}$ data points, a 4 s relaxation delay and 256 scans.

All 1D spectra were processed with a line broadening of 0.3 $\mathrm{Hz}$ and a zero filling factor of 2, manually phased and baseline corrected. The chemical shifts were referenced internally to the alanine signal at $\delta 1.48$ (this peak was found to be more reliable than the TSP peak due to the sensitivity of the latter to intermolecular interactions). 2D homonuclear and heteronuclear spectra were registered for selected samples to aid spectral assignment. These included total correlation spectroscopy (TOCSY), heteronuclear single quantum coherence (HSQC), and $J$-resolved spectroscopy experiments. Spectral assignment was carried out with the support of Bruker Biorefcode spectral database and specific compound standard solutions. In order to evaluate the magnitude of variation of some compounds, selected signals in the $1 \mathrm{D}$ spectra were integrated using the Amix-Viewer software (Bruker, version 3.9). Each area was normalized to total spectral area, excluding the water region, to enable comparison between samples. This approach does have some limitations since variations in metabolite absolute quantities may be masked and the assumption that total spectral area depends solely on the actual cell number in the rotor applies. Still, the method does enable reliable intersample comparison in terms of relative amounts of compounds.

Multivariate Analysis of NMR Spectral Data. A total of 40 spectra, corresponding to 16 control samples, $1230 \mu \mathrm{M}$ CDDPtreated samples and $1250 \mu \mathrm{M}$ CDDP-treated samples, have been considered for multivariate analysis. Data matrices were built from standard 1D spectra $(\delta 0.25-10)$, excluding the suppressed water signal $(\delta 4.9-5.1)$, and normalization was applied by adjusting the total area to unity. Then, after appropriate scaling (unit variance), Principal Component Analysis (PCA) and Partial Least Squares Regression Discriminant Analysis (PLS-DA) were applied to the data using Simca-P 11.5 (Umetrics, Sweden), employing a default 7-fold internal cross validation, from which $\mathrm{Q}^{2}$ and $\mathrm{R}^{2}$ values, representing, respectively, the predictive capability and the explained variance, were extracted. 
Statistical recoupling of variables (SRV) was performed on the data set according to the algorithm recently published. ${ }^{31}$ SRV is an unsupervised automated variable size bucketing procedure which focuses on the statistical relationships between consecutive variables inherited from the high-resolution bucketing. In this way, spectral dependency is restored by following a landscape of covariance/correlation $(L)$ between consecutive variables.

$$
L(i)=\frac{\text { covariance }}{\text { correlation }}(i, i+1)=\text { geometric mean }(i, i+1)
$$

Then the clusters whose borders are located at the minima of the covariance/correlation landscape are identified. These clusters must contain a minimum number of variables, which is defined as a function of the peak width of a singlet, at the baseline in the NMR spectrum, and bucketing resolution.

$$
\text { minimal no. variables }=\frac{\text { singlet base peak width }}{\text { bucketing resolution }}
$$

The next step of the procedure consists in the aggregation of neighboring clusters based on a sufficient threshold of cor-

a) control cells $0 \mathrm{~h}$
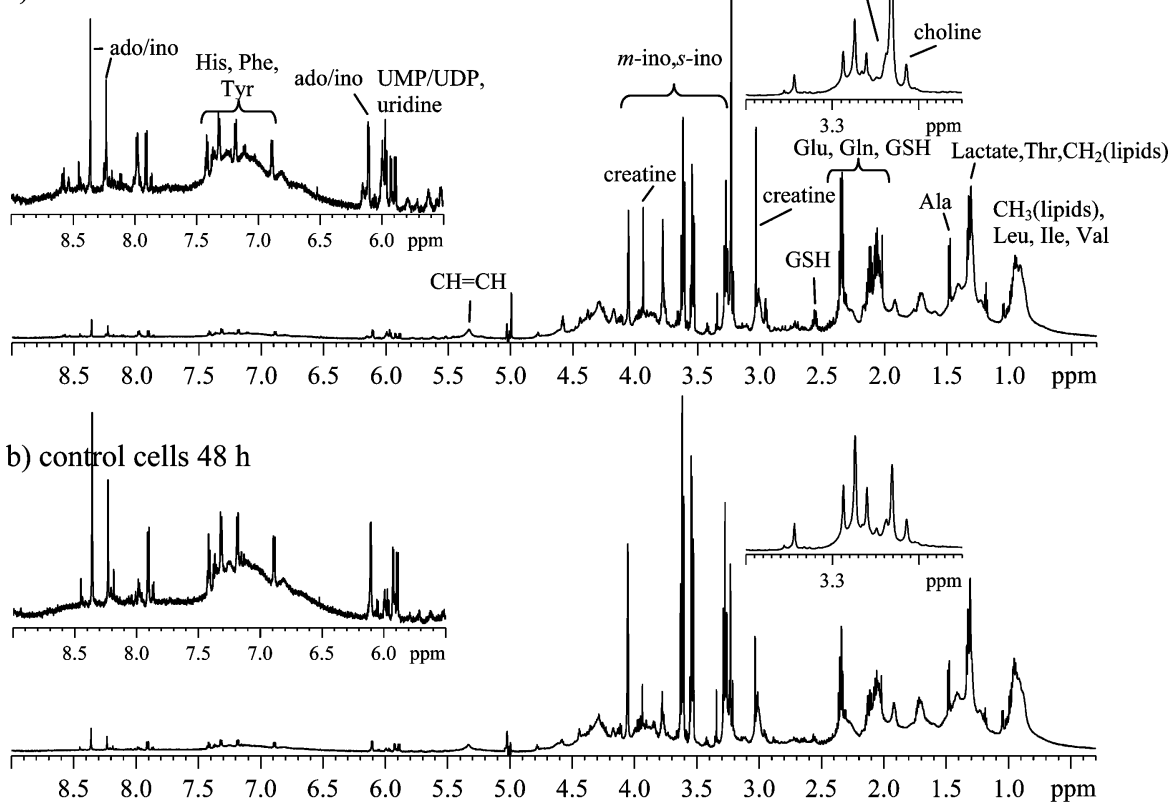

c) $30 \mu \mathrm{m}$ CDDP-treated cells $48 \mathrm{~h}$
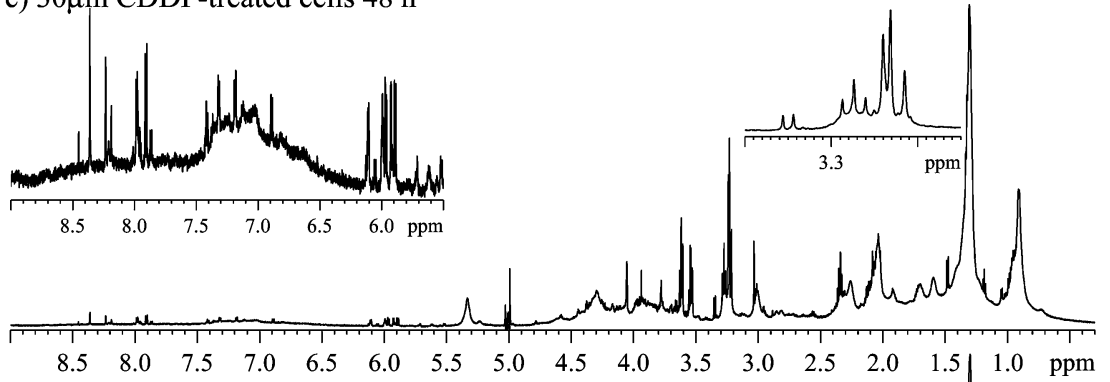

d) $50 \mu \mathrm{m}$ CDDP-treated cells $48 \mathrm{~h}$
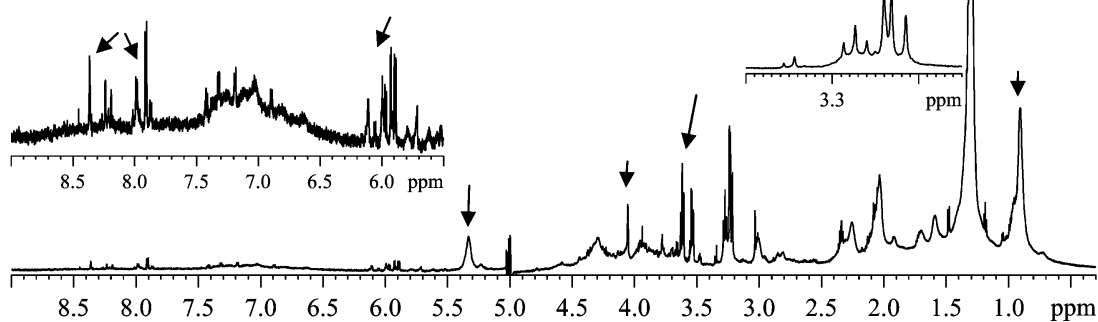

Figure 1. Average ${ }^{1} \mathrm{H}$ HRMAS Spectra Obtained for Control Cells at (a) 0 and (b) $48 \mathrm{~h}$; (c) $30 \mu \mathrm{m}$ CDDP-treated cells at $48 \mathrm{~h}$ and (d) 50 $\mu \mathrm{m}$ CDDP-treated cells at $48 \mathrm{~h}$. Arrows in (d) indicate visible changes compared to controls spectrum (b). Insets show expansions of the aromatic and 3.2-3.4 ppm regions. 
Table 1. Average Metabolite Variations Observed in Control and CDDP-Treated Cells $(30 \text { and } 50 \mu \mathrm{M})^{a}$

\begin{tabular}{|c|c|c|c|c|}
\hline Metabolite & $\delta{ }^{1} \mathrm{H}$ ppm (multiplicity) & Controls & CDDP $30 \mu \mathrm{M}$ & CDDP $50 \mu \mathrm{M}$ \\
\hline $\operatorname{Acetate}(\mathrm{s}) *$ & $2.02(\mathrm{~s})$ & $\downarrow(0-48 \mathrm{~h})$ & $\downarrow(0-48 \mathrm{~h})$ & $\downarrow(0-48 \mathrm{~h})$ \\
\hline Ado/Ino * & $4.44,4.78,6.10(\mathrm{~d}), 8.33(\mathrm{~s}), 8.36(\mathrm{~s})$ & $\begin{array}{l}\downarrow(0-18 \mathrm{~h}) \\
\uparrow(18-48 \mathrm{~h})\end{array}$ & $\downarrow(0-12 \mathrm{~h})$ & $\downarrow(0-12 \mathrm{~h})$ \\
\hline Choline (free)* & $3.21(\mathrm{~s})$ & - & $\begin{array}{l}\uparrow(0-18 \mathrm{~h}) \\
\downarrow(24-48 \mathrm{~h})\end{array}$ & $\begin{array}{l}\uparrow(0-18 \mathrm{~h}) \\
\downarrow(24-48 \mathrm{~h})\end{array}$ \\
\hline Creatine & $3.03(\mathrm{~s}), 3.94(\mathrm{~s})$ & $\downarrow(12-48 \mathrm{~h})$ & $\downarrow(0-48 \mathrm{~h})$ & $\downarrow(0-48 \mathrm{~h})$ \\
\hline GPC * & $3.24(\mathrm{~s}), 3.70$ & - & $\uparrow(12-48 \mathrm{~h})$ & $\uparrow(12-48 \mathrm{~h})$ \\
\hline GSH & $2.17,2.56,2.95,3.78,4.58$ & $\downarrow(0-48 \mathrm{~h})$ & $\downarrow(0-48 \mathrm{~h})$ & $\downarrow(0-48 \mathrm{~h})$ \\
\hline Lipids (fatty acyl chains) * & $0.90,1.30,1.59,2.25,2.83,5.33$ & - & $\uparrow(18-48 \mathrm{~h})$ & $\uparrow(18-48 \mathrm{~h})$ \\
\hline Lysine * & $1.73,1.92$ & $\uparrow(24-48 \mathrm{~h})$ & $\downarrow(24-48 \mathrm{~h})$ & $\downarrow(24-48 \mathrm{~h})$ \\
\hline myo-Inositol * & $3.27(\mathrm{t}), 3.54(\mathrm{dd}), 3.62(\mathrm{t}), 4.05(\mathrm{t})$ & $\uparrow(0-48 \mathrm{~h})$ & $\downarrow(24-48 \mathrm{~h})$ & $\downarrow(24-48 \mathrm{~h})$ \\
\hline $\mathrm{PC}$ & $3.23(\mathrm{~s}), 4.17$ & $\downarrow(0-48 \mathrm{~h})$ & $\downarrow(18-48 \mathrm{~h})$ & $\downarrow(18-48 \mathrm{~h})$ \\
\hline Tyrosine & $6.89,7.18$ & $\uparrow(24 \mathrm{~h})$ & - & $\downarrow(24-48 \mathrm{~h})$ \\
\hline UDP & $4.26,4.38,5.96(d), 5.99(d), 7.98$ (d) & $\downarrow(24-48 \mathrm{~h})$ & - & $\begin{array}{l}\uparrow(0-18 \mathrm{~h}) \\
\downarrow(18-48 \mathrm{~h})\end{array}$ \\
\hline Uridine & $4.14,4.23,4.36,5.89(\mathrm{~d}), 5.92(\mathrm{~d}), 7.91(\mathrm{~d})$ & $\uparrow(24-48 \mathrm{~h})$ & $\uparrow(24-48 \mathrm{~h})$ & $\uparrow(24-48 \mathrm{~h})$ \\
\hline Valine, Leucine *, Isoleucine & $0.93-1.06 \mathrm{ppm}$ (overlapped) & $\uparrow(24-48 \mathrm{~h})$ & - & $\downarrow(24-48 \mathrm{~h})$ \\
\hline
\end{tabular}

$a \uparrow$ increase, $\downarrow$ decrease, - no change (time period of variation in hours). Chemical shifts correspond to signals used to monitor each compound, not necessarily representing its complete spin system. ${ }^{*}$ compounds showing statistically relevant drug-specific variations. s: singlet, d: doublet, t: triplet, m: multiplet.

relation. This threshold is empirically determined by visualizing the identified clusters in a typical NMR spectrum of the data set. To set the threshold, the procedure is considered optimal when both strong aliphatic and weak aromatic multiplets are correctly identified. The clusters identified in this way then correspond to the physical, chemical or biological entities considered. It is noted that, in the case of two overlapping signals with different intensities, SRV usually identifies 3 clusters, with the central cluster corresponding to the weaker peak, and the two outer clusters corresponding to the stronger peak. This is notably observed for the broad lipid signals in HRMAS spectra, which may overlap with signals from small molecules. Thus, SRV does not discard the smaller peaks in overlapping regions, fuller details being given elsewhere. ${ }^{31}$ In this work, the following parameters have been used to efficiently recouple variables inherited from the high-resolution bucketing: 10 consecutive variables are required to describe a typical well-resolved singlet on a $700 \mathrm{MHz}$ spectrum (peak base width at the noise threshold for a resolved weak singlet in the aromatic area $=0.01 \mathrm{ppm}$, bucketing resolution $=0.001 \mathrm{ppm}$ ). The correlation threshold determined empirically by associating neighboring clusters along the chemical shift axis was 0.65. SRV was performed using Matlab R2008a (Mathworks, Natick, MA).

\section{Results}

Time Course Changes in MG-63 Cells in the Absence of Drug. Figure la shows the average ${ }^{1} \mathrm{H}$ HRMAS spectrum of control MG-63 cells, at $0 \mathrm{~h}$, indicating the importance of underlying broad signals arising from lipids and possibly proteins or other nitrogenated compounds, as well as a large number of resolved signals resulting from intracellular low molecular weight metabolites. Some signals are identified in the Figure, according to the detailed assignment previously reported for MG-63 cells. ${ }^{25}$ In the particular cell samples considered here, the following metabolites have been identified: organic acids (formate, fumarate, lactate), polyalcohols (myoand scyllo-inositols), several amino acids (Ala, Arg, Asp/Asn, Gln, Glu, Gly, His, Ile, Leu, Lys, Met, Phe, Pro, Thr, Tyr, Val) and amino acid derivatives (creatine, taurine and reduced glutathione - GSH), choline and choline-containing compounds (glycerophosphocholine, GPC; phosphocholine, PC), phosphoethanolamine (PE), nucleosides (cytidine and uridine) and nitrogenated bases adenosine/inosine (ado/iso). As seen before ${ }^{25}$ hardly any glucose is detected in the cells spectra, possibly due to its rapid intracellular conversion. Indeed, ${ }^{1} \mathrm{H}$ NMR spectra of culture media collected during the time of the experiment (not shown here) revealed a significant glucose uptake by the cells during the $48 \mathrm{~h}$ period.

Prior to the study of the effects of CDDP on the metabolome of MG-63 cells, the metabolic behavior of the untreated control cells over the experiment time was noted and, subsequently, taken as reference for the evaluation of the drug effects. Comparative inspection of the spectra of controls at 0 and $48 \mathrm{~h}$ (Figures 1a,b) enabled some variations to be identified, a more complete list of qualitative changes being shown in Table 1. These comprise decreases in acetate(s), creatine, glutamate, GSH, PC and UDP. In addition, increases are noted for myoand scyllo-inositols and, to lesser extents, for uridine and the amino acids Ala, Gly, Lys, Phe, Tyr and Val/Leu/Ile. For ado/ ino, more complex time variations are observed, with early decreases and subsequent increases at later stages.

Time Course Changes in MG-63 Cells Treated with Cisplatin (CDDP). The ${ }^{1} \mathrm{H}$ HRMAS average spectra of cells treated with 30 and $50 \mu \mathrm{M}$ CDDP, at $48 \mathrm{~h}$, are shown in Figure 1c,d, respectively. Comparison with the average control spectrum at $48 \mathrm{~h}$ (Figure 1b) enabled clear differences to be identified, some being slightly enhanced at the highest drug dosage. These differences relate, on one hand, to metabolites that show no 
a)

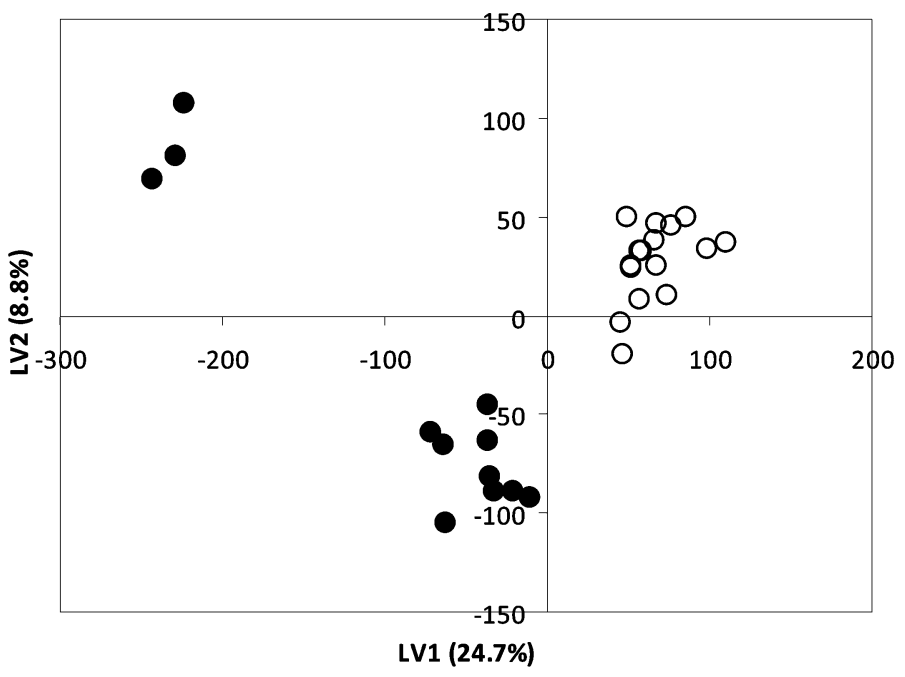

b)

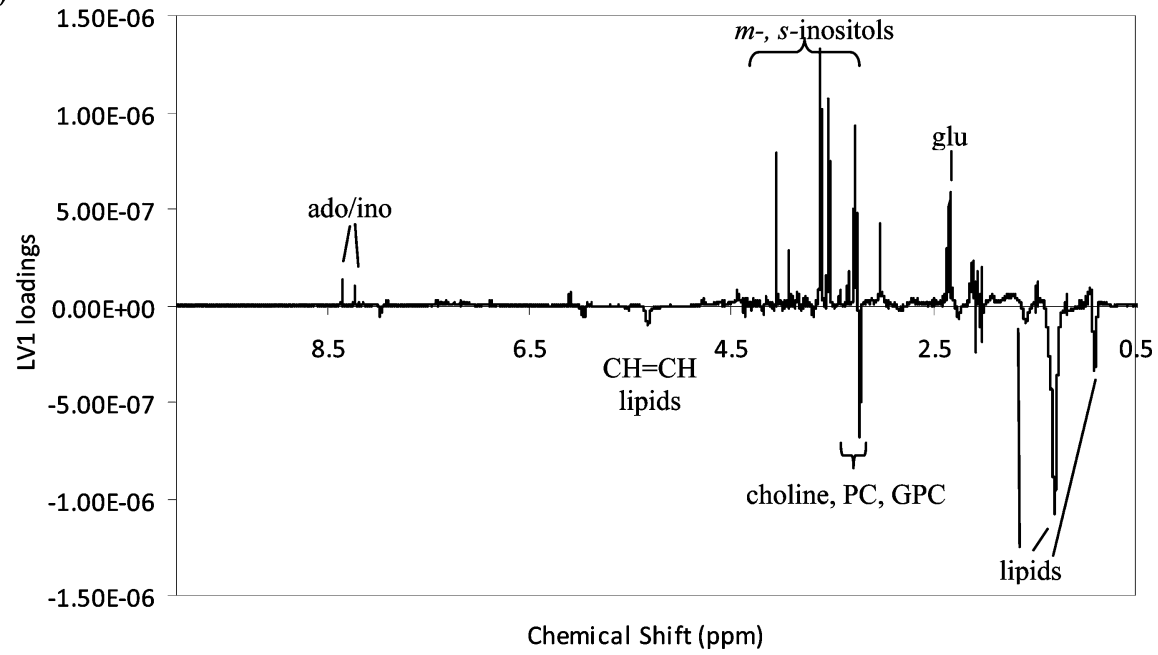

Figure 2. PLS-DA of controls $(O)$ and $50 \mu \mathrm{m}$ CDDP-treated cells $(\bullet)$, using the full spectral range ( 2 components calculated with $R^{2} \mathrm{X}=$ 0.34 and $Q^{2}=0.80$ ). (a) Scores scatter plot LV1 vs LV2; (b) LV1 loadings plot.

change whatsoever in the controls (free choline, GPC, lipids and taurine) and, on the other hand, to metabolites that exhibit altered time evolutions upon drug treatment compared to controls (ado/ino, inositols, UDP and amino acids Ala, Gly, Lys, Phe, Tyr and Val/Leu/Ile) (Table 1).

Multivariate analysis was applied to the cells spectra, in order to confirm visual observations and identify the most statistically relevant changes. PLS-DA of the spectra of controls and $50 \mu \mathrm{M}$ CDDP-treated cells reveals a clear discrimination between the two sample groups (Figure 2a), similar results having been observed for treatment with $30 \mu \mathrm{M}$ CDDP (not shown). The scores scatter plot in Figure 2a shows that all CDDP-treated cells are separated from controls along LV1, which explains $25 \%$ of the variability in the data. The corresponding loadings (Figure $2 \mathrm{~b}$ ) indicate that this separation is mainly due to increased lipids and choline compounds in treated cells (negative LV1 loadings), together with decreased levels of glutamate, myo-inositol and ado/ino (positive LV1 loadings), among others. Moreover, cells treated with CDDP for $48 \mathrm{~h}$ are further separated from the remaining treated cells along LV2, which explains $9 \%$ of the variability. LV2 loadings (not shown) suggest that samples treated with CDDP for $48 \mathrm{~h}$ are differentiated from treated samples collected at earlier times mainly based on lipid levels (higher at $48 \mathrm{~h}$ ) and on creatine, GSH, choline and PC contents (lower at $48 \mathrm{~h}$ ).

A more thorough interpretation of PLS-DA loadings may be achieved by SRV analysis, in order to identify the spectral changes with higher statistical relevance (or consistency across the samples universe). Such changes may be identified through color-coded plots such as that obtained for controls vs $50 \mu \mathrm{M}$ CDDP-treated cells (Figure 3). The colored LV1 loadings corresponding to the full spectrum (Figure 3a) readily show that the impact of CDDP treatment is reflected in all spectral regions, the peaks colored in red/orange identifying the varying metabolites with higher statistical importance for the separation between control and treated groups. In the plot in Figure 3a, the relevant peaks reflecting increased overall contents in CDDP-treated cells, compared to controls, are shown for negative covariances). These comprise lipid proton signals (methyl, $0.90 \mathrm{ppm}$; methylene, 1.30 and $1.59 \mathrm{ppm}$; and methine, $5.33 \mathrm{ppm})$, as well as free choline $(3.21 \mathrm{ppm})$ and GPC (3.24 ppm). Statistically relevant decreases (peaks with positive covariance) are seen to affect acetate (further reduced in treated cells vs controls), myo- and scylloinositols, ado/ino, amino acids Leu, Lys, Glu, Gly and taurine. The peak at $3.03 \mathrm{ppm}$ (Figure 3a) arises from the overlap of 


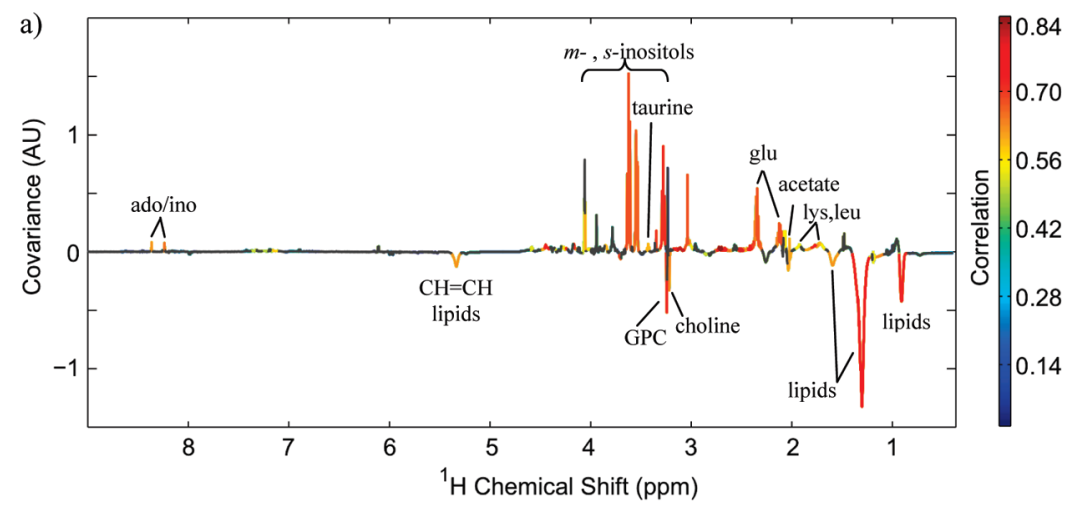

b)

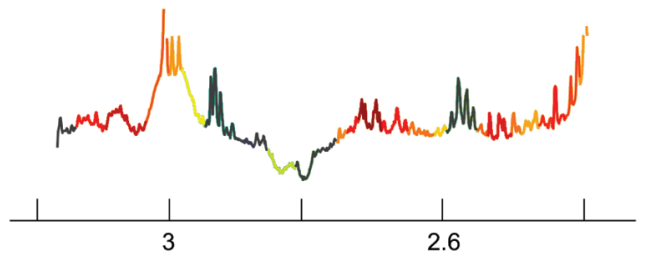

Figure 3. Statistical recoupling of variables (SRV) loadings (LV1) of (a) full spectra of control cells and $50 \mu \mathrm{m}$ CDDP-treated cells with color scale reflecting the statistical relevance of each signal; (b) the bottom plot shows the expansion of the 2.4-3.2 spectral region.

at least creatine and lysine, the latter being the most probable for the peak coloring, since the other creatine peak ( $\delta$ 3.94) has not been picked up by SRV. These relevant changes are noted with * in Table 1, lipids and 13 other compounds being selected as the most statistically relevant markers for MG-63 cells response to CDDP. Furthermore, SRV results enabled additional, still unassigned, spectral changes to be identified e.g. at 1.77, 2.4-3.2 ppm (expansion in Figure 3) and $4.45 \mathrm{ppm}$. Although unassigned, the SRV identification of these peaks shows the valuable potential of the NMR metabonomics approach in detecting exquisite and unexpected changes in the cellular metabolome.

Since the above MVA results only reflect the average behavior for each metabolite, thus not accounting for fine fluctuations in metabolite contents during the experiment, the main changing peaks were integrated whenever possible in order to more clearly evaluate the time-course drug-specific metabolite variations. These results are presented for controls and both drug doses, for lipids and choline compounds (Figure 4) and for selected amino acids, polyalcohols and nitrogenated bases (Figure 5). This semiquantitative analysis was carried out for all relevant metabolite changes (marked with * in Table 1), with the exception of acetate(s), Leu and Lys for which integration was hindered by severe spectral overlap. Furthermore, creatine was evaluated but confirmed not to vary significantly between controls and treated cells. The integrals confirm the clear increase in lipids $\left(\mathrm{CH}_{2}\right)_{n}$, compared to controls, from $12 \mathrm{~h}$, showing a more marked increase at the later stages (Figure $4 \mathrm{a}$ ). The fact that the $\left(\mathrm{CH}_{2}\right)_{n} / \mathrm{CH}_{3}$ ratio increases with time (Figure $4 \mathrm{~b}$ ) indicates that there is an average chain lengthening of the NMR-visible lipids over time, remaining higher than in controls throughout the experiment. In addition, the average unsaturation degree of visible lipids follows a similar trend (Figure 4c), as does the $5.23 \mathrm{ppm}$ resonance (not shown), reflecting the C2 glyceryl proton in phospholipids and triglycerides. The latter indicates that at least one of these classes is responsible for the noted lipid changes, nevertheless, concomitant fatty acids increase can not be ruled out and further characterization of the increasing lipids would require other analytical strategies, for example, lipid extract analysis. Regarding choline and choline-containing compounds, the effects are also clear in comparison to controls (Figure 4d,e,f). A marked increase is observed for GPC from $18 \mathrm{~h}$ (Figure 4f) and more complex trends are seen for free choline (Figure 4d) and PC (Figure 4e), with early accumulation followed by decrease back to control levels at $48 \mathrm{~h}$.

Additional changes relate to glutamate, which shows a small decrease relatively to controls (Figure 5a). Since this amino acid may relate closely to GSH, the content of the latter was monitored for comparison (not shown). However, GSH content was found to be very similar between drug-treated and control cells, with the exception of a relative decrease for treated cells at $48 \mathrm{~h}$. Other significant amino acid changes relate to glycine, which is relatively diminished from 24 to $48 \mathrm{~h}$ (Figure 5b) and taurine, which falls to lower contents from $18 \mathrm{~h}$ (Figure $5 \mathrm{c}$ ). In addition, lower contents of ado/ino (Figure $5 \mathrm{~d}$ ) and the inositol compounds (Figure 5e,f) consistently characterize the drugtreated cells, irrespective of drug dosage.

\section{Discussion}

Changes in Lipids and Choline Compounds. The lipid methyl, methylene and methine signals were seen to raise significantly in response to CDDP treatment, with hardly any change being noted for controls. In this respect, besides an increase in lipids content, increases in average aliphatic chain length and unsaturation degree were noted. Several possible origins have been suggested for the lipid signals visible in the NMR spectrum, namely lipid droplets in the cytosol, or membrane lipids in the form of glycerophospholipids ${ }^{15,16}$ or triglycerides microdomains embedded in the plasma membrane. ${ }^{28}$ In several types of tumor cells, the enhancement of these lipid signals, as well as of the unsaturion degree, has been consistently noted and correlated to cells undergoing apoptosis, this effect having been observed for instance in MG-63 cells undergoing radiation-induced apoptosis. ${ }^{11}$ A lengthening of the 
a)

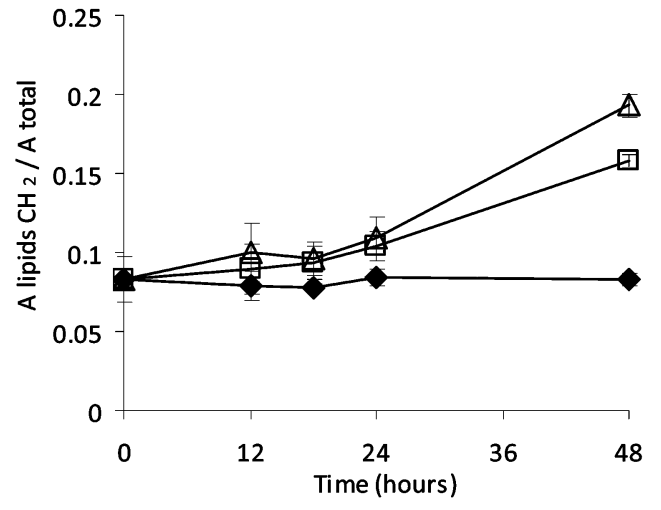

c)

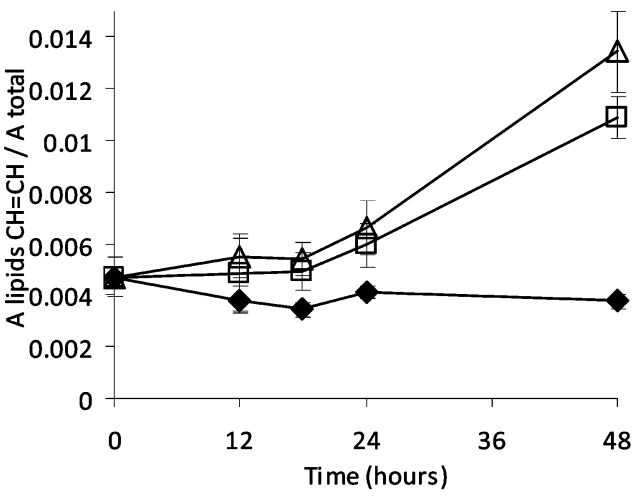

e)

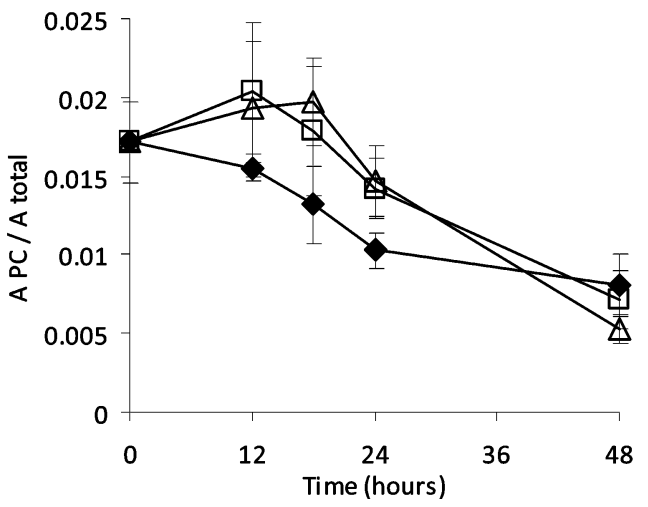

b)

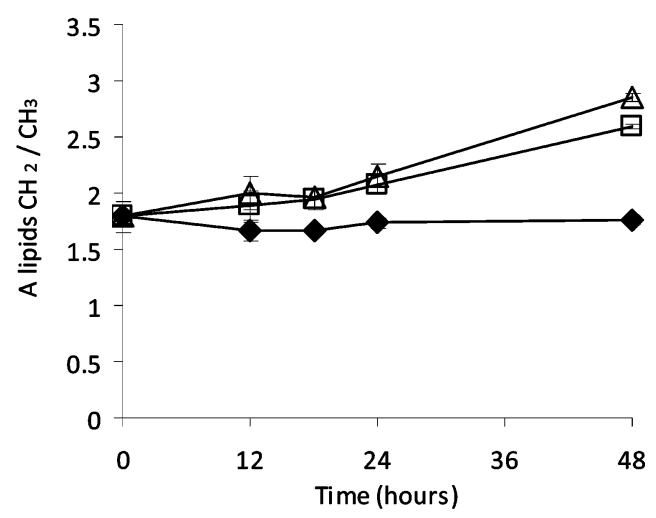

d)

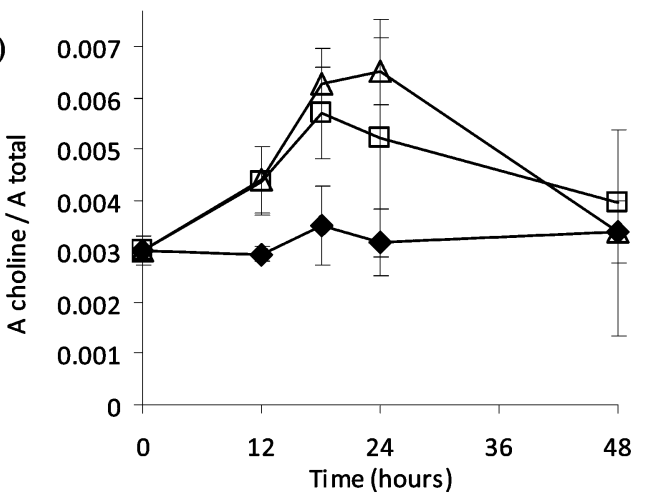

f)

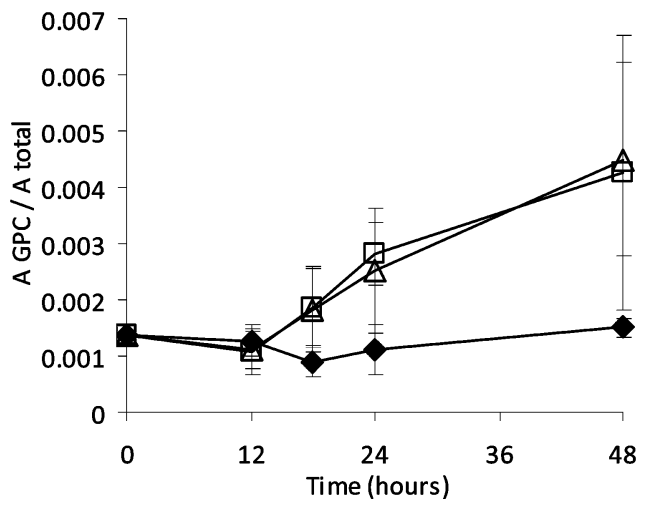

Figure 4. Plots of area ratios (to total spectral area) for (a) lipids $\left(\mathrm{CH}_{2}\right)_{n}$, (b) lipids $\left(\mathrm{CH}_{2}\right)_{n} / \mathrm{CH}_{3}$, (c) lipids $\mathrm{CH}=\mathrm{CH}$, (d) choline, (e) phosphocholine, PC and (f) glycerophosphocholine, GPC, during CDDP exposure time. Control cells ( $\diamond), 30 \mu \mathrm{m}$ CDDP-treated cells $(\square)$ and $50 \mu \mathrm{m}$ CDDP-treated cells $(\triangle)$.

fatty acid $\mathrm{CH}_{2}$ chain has also been associated with apoptosis in cervical carcinomas. ${ }^{32}$ In this work, the effect of CDDP on lipids is visible from the initial stages but more marked from 24 to $48 \mathrm{~h}$, suggesting increased cell apoptosis at this later stage. As to the reason why the accumulated lipids in CDDP-treated cells tend to have longer chains and higher unsaturation degrees, it remains, to our knowledge, unclear.

Regarding free choline and choline-containing compounds PC and GPC, the effect of the drug is also clear. Since GPC is solely a breakdown product of membrane phosphatidylcholine, $\mathrm{PTC}^{33}$ its clear increase compared to controls (Figure 4f) suggests increasing membrane degradation from $18 \mathrm{~h}$ of CDDP exposure. In turn, GPC degrades into free choline, which should contribute to the enhanced choline peak at 18 and $24 \mathrm{~h}$ (Figure $4 \mathrm{~d})$. However, an enhanced choline content is already observed before, at $12 \mathrm{~h}$, which suggests the contribution of other effects not involving the formation of GPC, such as the activation of other PTC degradative pathways. ${ }^{33}$ At $48 \mathrm{~h}$, choline content decreases back to controls level, very much in parallel to PC variation at the latest stage of drug exposure (Figure 4e), suggesting a possible metabolic relationship between these compounds for longer exposures. PC levels are known to be increased in tumoral cells (compared to nontumoral ones) due to several concomitant effects: increased phospholipid biosynthesis to meet cell proliferation requirements, activation of PTC-cycle enzymes and increased choline transport into the cell. ${ }^{4}$ In MG-63 control cells, PC shows a steady decrease over time (Figure 4e), suggesting the occurrence of time-dependent counter effects which, however, leave choline levels unaltered (Figure 4d). These may involve changes in phospholipid biosynthesis or PTC-cycle regulation, rather than increased choline uptake, since the particular cell media employed here 
a)

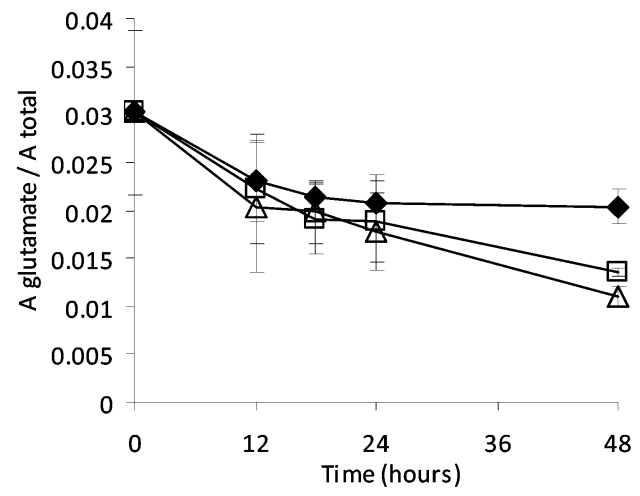

c)

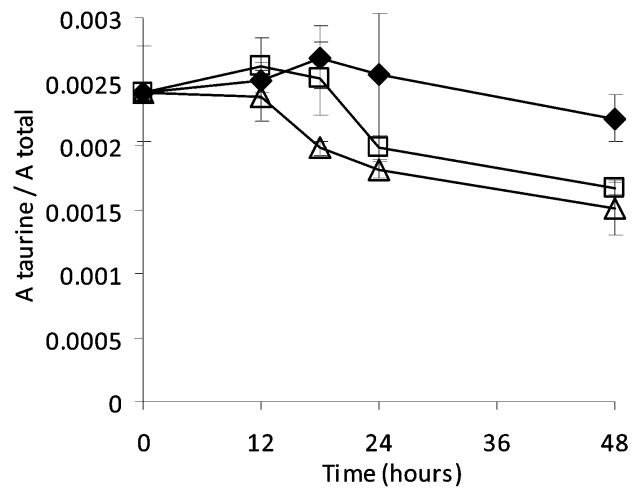

e)

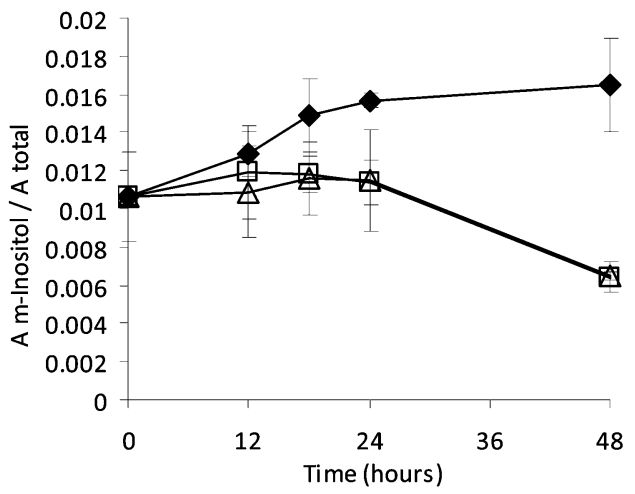

b)

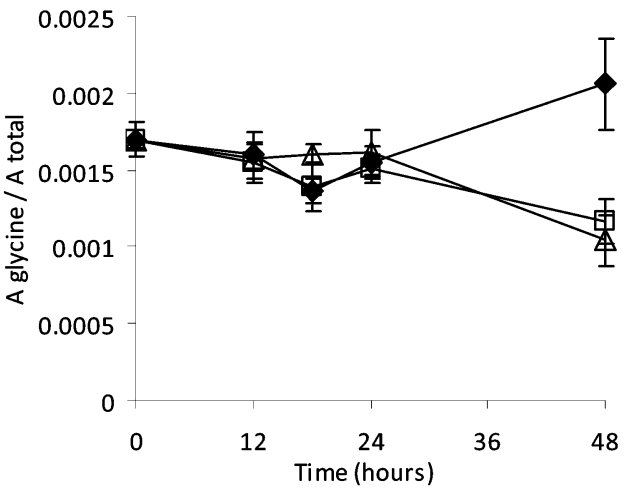

d)

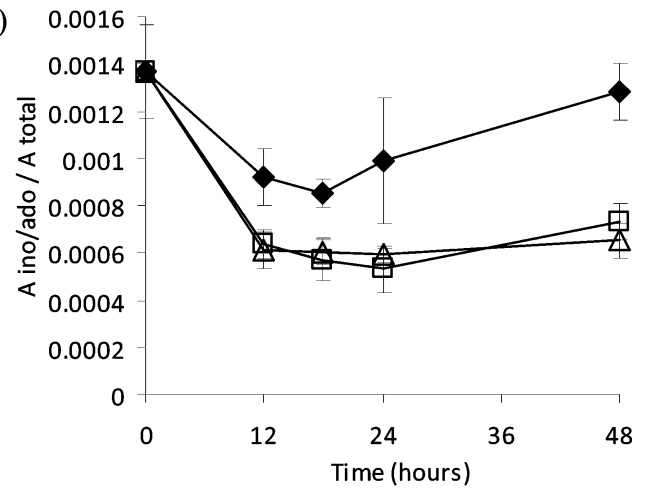

f)

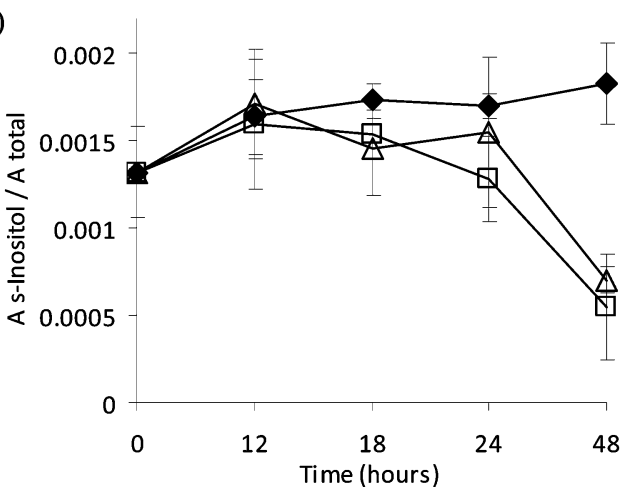

Figure 5. Plots of area ratios (to total spectral area) for (a) glutamate, (b) glycine, (c) taurine, (d) ado/ino, (e) myo-, and (f) scylloinositol, during CDDP exposure time. Control cells $(\diamond), 30 \mu \mathrm{m}$ CDDP-treated cells $(\square)$, and $50 \mu \mathrm{m}$ CDDP-treated cells $(\Delta)$.

does not contain this metabolite. In the presence of CDDP, however, PC accumulates up to $24 \mathrm{~h}$, which may relate, not to increased cell proliferation but, again, to PC and PTC enzyme regulation changes promoting the use of intracellular choline. From 18 h onward, PC levels decrease back to control levels which may reflect the eventual shortage of choline in the cell, at later stages.

Changes in Other Metabolites. Glutamate shows a marked decrease in CDDP-treated cells (Figure 5a), both compared to controls (particularly at $48 \mathrm{~h}$ ) and over time. This metabolite is an important transamination partner, being readily converted into $\alpha$-ketoglutarate, thus acting as an anaplerotic agent to the Krebs cycle. A glutamate decrease may reflect an increase in mitochondrial activity in the presence of CDDP. This metabolite is also an important precursor of GSH, which is known to be related to the cell's defense mechanisms toward oxidative stress. It is recalled that GSH shows, indeed, a relative decrease over time, particularly noted for CDDP-treated cells at $48 \mathrm{~h}$. It is possible, therefore, that the glutamate variation observed is also determined by the effects of oxidative stress over time, more enhanced in the presence of CDDP, consistently with other recent findings for drug-induced metabolic changes in tumoral cells. ${ }^{22}$ The oxidized form of glutathione (GSSG) would then be expected to accumulate, giving characteristic NMR signals in the 2-4 ppm region, however, although some weak changes are indeed observed in this region (expansion in Figure 3), no clear evidence of GSSG increase has been found.

The decrease in taurine (Figure 5b) is consistent with previously reported observations relating to MG-63 cells subjected to radiation. ${ }^{11}$ The antioxidant properties of this compound have been related to the cell's defense mechanism of DNA protection toward oxidative effects. The marked decreases in myo- and scyllo-inositol may be related to the osmoregulatory roles of these compounds in the cell. An increase in the former compound has been observed in some tumor cells, as 
a response to cell proliferation increase ${ }^{4}$ whereas a decrease has been noted in myeloid leukemia upon exposure to specific drugs. 22

Finally, the significant ado/ino decrease may reflect reduced DNA synthesis as a result of the CDDP action through DNA cross-linking and subsequent double-strand breakage. Other important metabolite changes comprise acetate(s) and the amino acids Gly, Lys and Leu, although their relevance remains unclear without further specific studies, such as specific nutrient labeling assays.

\section{Conclusions}

In the present study, ${ }^{1} \mathrm{H}$ HRMAS NMR spectroscopy was used to assess the changes in the intracellular metabolic profile of MG-63 human OS cells induced by the chemotherapy agent cisplatin (CDDP), over a period of up to $48 \mathrm{~h}$. Many changes were clearly observed in the NMR spectra and, indeed, consistent spectral variations were unveiled by PLS-DA of the spectra. All CDDP-treated cells were clearly separated from the controls, the $48 \mathrm{~h}$ samples being further separated from the remaining treated cells. Indeed, with basis on SRV analysis and spectral integration of some peaks, it became apparent that the main metabolite changes were more marked at the later stage of drug exposure (24-48 h). Lipids, choline and cholinecontaining compounds were some of the main players in MG63 cell response to CDDP, suggesting the occurrence of apoptosis and alterations in lipid metabolism regulation. In addition, some amino acids were significantly changed, the decrease in Glu and taurine possibly reflecting effects of oxidative stress overtime and activation of cell DNA-related defense mechanisms, respectively. Significant reductions in osmoregulatory compounds myo- and scyllo-inositols indicated their role in cellular stress response whereas the decreasing nitrogenated bases ado/ino could reflect reduced DNA synthesis. Further interpretation of these metabolic changes will entail, in future studies, the use of stable-isotope labeled nutrients and selected enzymatic analysis.

Acknowledgment. Funding is acknowledged from Fundação para a Ciência e Tecnologia (FCT) in Portugal (FCT/PTDC/SAU-BEB-66896/2006 and FCT/SFRH/BD/63916/ 2009), the Associate Laboratory IBMC-INEB (for kindly providing the MG-63 cell line) and CRMN- Centre de RMN à Très Hauts Champs, CNRS/ENS Lyon, France, for access to the $700 \mathrm{MHz}$ NMR spectrometer. B.J.B. acknowledges support from Bruker Biospin, the French National Institute for Cancer INCa (Grant INCa-2008-060) and the CNRS. Financial support by the Access to Research Infrastructures activity in the sixth Framework Programme of the EC (Contract \# RII3-026145, EU-NMR) for conducting the research is gratefully acknowledged.

\section{References}

(1) Chou, A. J.; Geller, D. S.; Gorlick, R. Therapy for osteosarcoma: where do we go from here. Pediatric Drugs 2008, 10 (5), 315-327.

(2) Chou, A. J.; Gorlick, R. Chemotherapy resistance in osteosarcoma: current challenges and future directions. Expert Rev. Anticancer Ther. 2006, 6 (7), 1075-1085.

(3) Chung, Y. L.; Griffiths, J. R. Using metabonomics to monitor anticancer drugs, in Oncogenes Meet Metabolism; Spinger-Verlag: Berlin, 2008; pp 55-78.

(4) Merz, A. L.; Serkova, N. J. Use of nuclear magnetic resonancebased matabolomics in detecting drug resistance in cancer. Biomarkers Med. 2009, 3 (3), 289-306.
(5) Griffin, J. L.; Shockcor, J. P. Metabolic profiles of cancer cells. Nat. Rev. Cancer 2004, 4, 551-561.

(6) Lutz, N. W. From metabolic to metabolomic NMR spectroscopy of apoptotic cells. Metabolomics 2005, 1 (3), 251-268.

(7) Khoo, S. H. G.; Al-Rubeai, M. Metabolomics as a complementary tool in cell culture. Biotechnol. Appl. Biochem. 2007, 47, 71-84.

(8) Duarte, I. F.; Lamego, I.; Rocha, C.; Gil, A. M. NMR metabonomics for mammalian cell metabolism studies. Bioanalysis 2009, 1 (9), 1597-1614.

(9) Santini, M. T.; Rainaldi, G.; Romano, R.; Ferrante, A.; Clemente, S.; Motta, A.; Indovina, P. L. MG-63 human osteosarcoma cells grown in monolayer and as three-dimentional tumor spheroids present a different metabolic profile: a 1H NMR study. FEBS Lett. 2004, 557, 148-154.

(10) Santini, M. T.; Romano, R.; Rainaldi, G.; Ferrante, A.; Motta, A.; indovina, P. L. Increases in 1H-NMR mobile lipids are not always associated with overt apoptosis: evidence from MG-63 human osteosarcoma three-dimensional spheroids exposed to a low dose (2 Gy) of ionizing radiation. Radiat. Res. 2006, 165, 131-141.

(11) Santini, M. T.; Romano, R.; Rainaldi, G.; Indovina, P.; Ferrante, A.; Motta, A.; Indovina, P. L. Temporal dynamics of $1 \mathrm{H}-\mathrm{NMR}-$ visible metabolites during radiation-induced Aapoptosis in MG63 human osteosarcoma Spheroids. Radiat. Res. 2006, 166, 734745.

(12) Santini, M. T.; Romano, R.; Rainaldi, G.; Ferrante, A.; Indovina, P.; Motta, A.; Indovina, P. L. 1H-NMR evidence for a different response to the same dose (2 Gy) of ionizing radiation of MG-63 human osteosarcoma cells and three-dimensional spheroids. Anticancer Res. 2006, 26 (1A), 267-281.

(13) Loiseau, D.; Morvan, D.; Chevrollier, A.; Demidem, A.; Douay, O.; Reynier, P.; Stepien, G. Mitochondrial bioenergetic background confers a survival advantage to HepG2 cells in response to chemotherapy. Mol. Carcinogenesis 2009, 48, 733-741.

(14) Cooper, W. A.; Bartier, W. A.; Rideout, D. C.; Delikatny, E. J. 1H NMR visible lipids are induced by phosphonium salts and 5-fluorouracil human breast cancer cells. Magn. Reson. Med. 2001, 45, 1001-1010.

(15) Brisdelli, F.; Iorio, E.; Knijn, A.; Ferretti, A.; Marcheggiani, D.; Lentie, L.; Strom, R. Two-step formation of $1 \mathrm{H}$ NMR visible mobile lipids during apoptosis of paclitaxel-treated K562 cells. Biochem. Pharmacol. 2003, 65, 1271-1280.

(16) Sathasivam, N.; Brammah, S.; Wright, L. C.; Delikatny, E. Inhibition of tetraphenylphosphonium-induced NMR-visible lipid accumulation in human breast cells by chlorpromazine. Biochim. Biophys. Acta 2003, 1633, 149-160.

(17) Knijn, A.; Brisdelli, F.; Ferretti, A.; Iorio, E.; Marcheggiani, D.; Bozzi, A. Metabolic alterations in K562 cells exposed to taxol and tyrphostin AG957: $1 \mathrm{H}$ NMR and biochemical studies. Cell Biol. Int. 2005, 29, 890-897.

(18) Al-Saffar, N. M. S.; Troy, H.; de Molina, A. R.; Jackson, L. E.; Madhu, B.; Griffiths, J. R.; Leach, M. O.; Workman, P.; Lacal, J. C.; Judson, I. R.; Chung, Y.-L. Noninvasive magnetic resonance spectroscopic pharmacodynamic markers of the choline kinase inhibitor MN58b in human carcinoma models. Cancer Res. 2006, 66 (1), 427-434.

(19) Fan, T. W.-M.; Higashi, R. M.; Lane, A. N. Integrating metabolomics and transcriptomics for probing Se anticancer mechanisms. Drug Metab. Rev. 2006, 38, 707-732.

(20) Morse, D. L.; Raghunand, N.; Sadarangani, P.; Murthi, S.; Job, C.; Day, S.; Howison, C.; Gillies, R. J. Response of choline metabolites to docetaxel therapy is quantified in vivo by localized 31P MRS of human breast cancer xenografts and in vitro by high-resolution 31P NMR spectroscopy of cell extracts. Magn. Reson. Med. 2007, $58,270-280$.

(21) Kominsky, D. J.; Klawitter, J.; Brown, J. L.; Boros, L. G.; Melo, J. V.; Eckhardt, S. G.; Serkova, N. Abnormalities in glucose uptake and metabolism in imatinib-resistant human BCR-ABL-positive cells. Clin. Cancer Res. 2009, 15 (10), 3442-3450.

(22) Tiziani, S.; Lodi, A.; Khanim, F. L.; Viant, M. R.; Bunce, C. M.; Günter, U. L. Metabolomic profiling of drug responses in acute myeloid leukaemia cell lines. PLOS One 2009, 4 (1), 1-10.

(23) Lindon, J. C.; Beckonert, O. P.; Holmes, E.; Nicholson, J. Highresolution magic angle spinning NMR spectroscopy: Application to biomedical studies. Prog. Nucl. Magn. Reson. Spectrosc. 2009, 55 (2), 79-100.

(24) Sitter, B.; Bathen, T. F.; Tessem, M.-B.; Gribbestad, I. S. Highresolution magic angle spinning (HR MAS) MR spectroscopy in metabolic characterization of human cancer. Prog. Nucl. Magn. Reson. Spectrosc. 2009.

(25) Duarte, I. F.; Marques, J.; Ladeirinha, A. F.; Rocha, C.; Lamego, I.; Calheiros, R.; Silva, T. M.; Marques, M. P. m.; Melo, J. B.; Carreira, I. M.; Gil, A. M. Analytical approaches toward successful human 
cell metabolome studies by NMR spectroscopy. Anal. Chem. 2009, 81, 5023-5032.

(26) Griffin, J. L.; Pole, J. C. M.; Nicholson, J. K.; Carmichael, P. L. Cellular environment of metabolites and a metabonomic study of tamoxifen in endometrial cells using gradient high resolution magic angle spinning 1H NMR spectroscopy. Biochim. Biophys. Acta 2003, 1619, 151-158.

(27) Morvan, D.; Demidem, A.; Papon, J.; Madelmont, J. C. Quantitative HRMAS proton total correlation spectroscopy applied to cultured melanoma cells treated by chloroethyl nitrosourea: demonstration of phospholipid metabolism alterations. Magn. Reson. Med. 2003, 49, 241-248.

(28) Borel, M.; Degoul, F.; Communal, Y.; Mounetou, E.; Bouchon, B.; C-Gaudreault, R.; Madelmont, J. C.; Miot-Noirault, E. N-(4iodophenyl)-N0-(2-chloroethyl)urea as a microtubule disrupter: in vitro and in vivo profiling of antitumoral activity on CT-26 murine colon carcinoma cell line cultured and grafted to mice. $\mathrm{Br}$. J. Cancer 2007, 96, 1684-1691.
(29) Durkin, W. S.; Ghanta, V. K.; Balch, C. M.; Davis, D. W.; Hiramoto, R. N. A methodological approach to the prediction of anticancer drug effects in humans. Cancer Res. 1979, 39, 402-407.

(30) Li, Y. L.; Lin, A. W.; Zhang, X. J.; Wang, Y. Q.; Wang, X. L.; Goodrich, D. W. Cancer cells and normal cells differ in their requirements for Thoc1. Cancer Res. 2007, 67, 6657-6664.

(31) Blaise, B. J.; Shintu, L.; Elena, B.; Emsley, L.; Dumas, M.-E.; Toulhoat, P. Statistical recoupling prior to significance testing in nuclear magnetic resonance based metabonomics. Anal. Chem. 2009, 81, 6242-6251.

(32) Lyng, H.; Sitter, B.; Bathen, T. F.; Jensen, L. R.; Sundfør, K.; Kristensen, G. B.; Gribbestad, I. S. Metabolic mapping by use of high-resolution magic angle spinning $1 \mathrm{H}$ MR spectroscopy for assessment of apoptosis in cervical carcinomas. BMC Cancer 2007, $7,11$.

(33) Glunde, K.; Jacobs, M. A.; Pathaka, A. P.; Artemov, D.; Bhujwalla, Z. M. Molecular and functional imaging of breast cancer. NMR Biomed. 2009, 22 (1), 92-103.

PR100635N 